REVISTA DE GEOCIÊNCIAS DO NORDESTE

Northeast Geosciences Journal

v. $6, n^{\circ} 2(2020)$

https://doi.org/10.21680/2447-3359.2020v6n2ID19111

ISSN: $2447-3359$

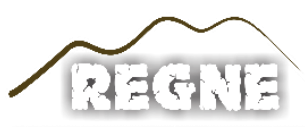

\title{
ESTIMATIVA DA VULNERABILIDADE NATURAL À CONTAMINAÇÃO DO AQUÍFERO EM ESCALA MUNICIPAL: ESTUDO DE CASO EM PANAMBI/RS
}

\begin{abstract}
Gabriel D'ávila Fernandes ${ }^{1}$; Willian Fernando de Borba $^{2}$; Éricklis Edson Boito de Souza ${ }^{3}$; Pedro Daniel da Cunha Kemerich ${ }^{4}$; José Luiz Silvério da Silva ${ }^{5}$; Lêonidas Luiz Volcato Descovi Filho ${ }^{6}$; Diego Hinterholz ${ }^{7}$; Edner Baumhardt ${ }^{8}$
\end{abstract}

${ }^{1}$ Doutorando em Engenharia Civil, Programa de Pós-Graduação em Engenharia Civil, Universidade Federal de Santa Maria (UFSM), Santa Maria/RS, Brasil.

ORCID: https://orcid.org/0000-0002-1106-3838

Email: enggabrielfernandes@gmail.com

${ }^{2}$ Doutor em Engenharia Civil, Departamento de Engenharia e Tecnologia Ambiental, Universidade Federal de Santa Maria (UFSM), Frederico Westphalen/RS, Brasil.

ORCID: https://orcid.org/0000-0001-5717-1378

Email: borbawf@gmail.com

${ }^{3}$ Mestrando em Engenharia Florestal, Programa de PósGraduação em Engenharia Florestal, Universidade Federal de Santa Maria (UFSM), Santa Maria/RS, Brasil.

ORCID: $\underline{\text { https://orcid.org/0000-0001-8138-8040 }}$

Email: ericklisboito@gmail.com

${ }^{4}$ Doutor em Engenharia Ambiental, Pró-Reitoria de Graduação, Universidade Federal do Pampa (UNIPAMPA), Bagé/RS, Brasil. ORCID: https://orcid.org/0000-0002-9369-769X

Email: eng.kemerich@yahoo.com.br

${ }^{5}$ Doutor em Geociências, Departamento de Geociências, Universidade Federal de Santa Maria (UFSM), Santa Maria/RS, Brasil.

ORCID: https://orcid.org/0000-0003-1712-9145

Email: silverioufsm@gmail.com

${ }^{6}$ Doutor em Geografia, Instituto de Engenharia e Geociências, Universidade Federal do Oeste do Pará (UFOPA), Santarém/PA, Brasil.

ORCID: https://orcid.org/0000-0001-9245-308X

Email: leonprs@gmail.com

${ }^{7}$ Engenheiro Ambiental e Sanitarista, Universidade Federal de Santa Maria (UFSM), Frederico Westphalen/RS, Brasil.
ORCID: https://orcid.org/0000-0003-1776-0922

Email: diegohinterholz@ hotmail.com

${ }^{8}$ Doutor em Engenharia Florestal, Departamento de Engenharia Florestal, Universidade Federal de Santa Maria (UFSM), Frederico Westphalen/RS, Brasil.

ORCID: $\underline{\text { https://orcid.org/0000-0001-8480-4521 }}$

Email: ednerb@gmail.com

\section{Resumo}

A avaliação da vulnerabilidade de aquíferos é uma ferramenta importante para avaliar a contaminação das águas subterrâneas. O emprego de técnicas inadequadas do preparo do solo e o crescimento populacional ocasionam severas complicações ambientais, indagando a correta gestão de recursos hídricos, sejam superficiais ou subterrâneos. Essa pesquisa teve como objetivo avaliar localmente a vulnerabilidade natural à contaminação do Sistema Aquífero Serra Geral em Panambi, localizado no noroeste do estado do Rio Grande do Sul. Os resultados demonstraram que o aquífero, em sua maior parte, apresentou classes de vulnerabilidade que variaram de insignificante à baixa. Entretanto, na área são realizadas atividades com potencial poluidor, como agricultura intensiva, além do polo metal mecânico, que podem alterar a qualidade do meio subterrâneo, caso ocorra negligência de normativas ambientais.

Palavras-chave: Água subterrânea; Gestão territorial; SASG.

ESTIMATES OF NATURAL VULNERABILITY SET FOR AQUIFER CONTAMINATION AT MUNICIPAL SCALE: A CASE STUDY IN PANAMBI (RIO GRANDE DO SUL STATE/BRAZIL)

Abstract
Vulnerability assessment applied to aquifers is an important tool
to assess contamination in this environment. Inappropriate soil
tillage techniques and population growth cause severe
environmental complications and account for the need of
adopting correct management for surface water and groundwater
resources. The aim of the current research is to assess natural
vulnerability to contamination in the Serra Geral Aquifer System
through the GOD methodology in Panambi County, Northwest


Rio Grande do Sul State - Brazil. Results have shown that the aquifer presented vulnerability classes ranging from insignificant to low. However, there are activities with polluting potential in the area, such as intensive agriculture, in addition to the mechanical metal industries hub; these activities, which can change the quality of the underground environment in case environmental standards are neglected.

Keywords: Groundwater; Territorial management; SASG.

\section{ESTIMACIÓN DE VULNERABILIDAD NATURAL A LA CONTAMINACIÓN DEL ACUÍFERO A ESCALA MUNICIPAL: ESTUDIO DE CASO EN PANAMBI (RIO GRANDE DO SUL/BRAZIL)}

\section{Resumen}

La evaluación de la vulnerabilidad de los acuíferos es una herramienta importante para evaluar la contaminación del agua subterránea. El uso de técnicas inadecuadas de preparación del suelo y el crecimiento poblacional provocan graves complicaciones ambientales, cuestionando la correcta gestión de los recursos hídricos, ya sean superficiales o subterráneos. Esta investigación tuvo como objetivo evaluar localmente la vulnerabilidad natural a la contaminación del Sistema Acuífero Serra Geral en Panambi, ubicado en el noroeste del estado de Rio Grande do Sul - Brazil. Los resultados mostraron que el acuífero, en su mayor parte, presenta clases de vulnerabilidad que varían de insignificante a baja. Sin embargo, en la zona se realizan actividades con potencial contaminante, como la agricultura intensiva, además del poste metálico mecánico, que pueden alterar la calidad del medio subterráneo, en caso de incumplimiento de la normativa ambiental.

Palabras-clave: Agua subterrânea; Gestión territorial; SASG.

\section{INTRODUÇÃO}

A contaminação dos recursos hídricos superficiais ocasionada principalmente pelo crescimento demográfico, aliada à falta de obras de infraestrutura (sistemas de coleta e tratamento de esgotos, por exemplo) é um grave problema que afeta muitos municípios do Brasil. Com isso, o abastecimento a partir de fontes alternativas é uma opção atraente para sanar esse grave impasse.

A água subterrânea é uma alternativa para suprir as necessidades da população principalmente para regiões de difícil acesso, ou seja, onde não se tem rede de água disponível. Isso pode ocorrer em núcleos populacionais nas áreas rurais dos municípios, sendo o uso da água subterrânea muito comum atualmente. Porém, senão ocorrer a gestão adequada desse recurso hídrico, isso pode acarretar sérios problemas ambientais, relacionados principalmente a disponibilidade desse recurso. Freeze e Cherry (2017) dizem que a qualidade da água subterrânea vem sendo degradada com o passar do tempo.

$\mathrm{O}$ uso das geotecnologias possibilitou o melhor conhecimento dos recursos naturais (água, solo e vegetação), desta forma, temse uma melhoria na avaliação do potencial da exploração de terras sendo desenvolvida de forma mais sustentável (FRANCISCO et al., 2018). Além disso, os mesmos autores afirmam que essas ferramentas auxiliam no desenvolvimento regional planejado.
Encina et al. (2018) citam que os dados das geotecnologias podem ser anexados em bancos de dados georreferenciados.

Alguns métodos utilizados para auxiliar na gestão dos recursos hídricos seriam estimando a vulnerabilidade natural à contaminação do aquífero. Dentre os vários existentes se tem o sistema GOD (Groundwater hydraulic confinement, Overlaying strata, Depth to groundwater table), desenvolvido por Foster et al. (2002; 2006), o método DRASTIC (Depth to the water, Net Recharge, Aquifer material, Soil Type, Topografy, Impact of the unsatured zone, Hydraulic Conductivity), proposto por Aller et al. (1987) e o Índice de Susceptibilidade (Ribeiro, 2005).

O sistema GOD apresenta bons resultados para condições brasileiras e caribenhas (Foster et al., 2002; 2006), sendo amplamente utilizado tanto em rochas vulcânicas como em rochas sedimentares. Dentre os muitos estudos realizados no Brasil, destacam-se principalmente as pesquisas de Reginato e Alhert (2013), Nanni et al. (2005), Cutrim e Campos (2010), onde foram estimados o índice de vulnerabilidade natural do aquífero à contaminação.

Atividades potencialmente poluidoras, realizadas na superfície de locais com características do meio que facilitem a percolação desses contaminantes, expõem a água subterrânea ao risco de que seja atingida por esses contaminantes (Foster et al. 2002; 2006). O município de Panambi - RS, localizado no planalto sul-riograndense, destaca-se não somente no cenário regional, mas nacional e internacional, como um polo metal mecânico, com a presença das mais variadas indústrias desse setor.

Com isso, estudos que utilizam a temática envolvendo a estimativa da vulnerabilidade do aquífero são de suma importância, pois irão elencar quais as áreas mais vulneráveis à contaminação por atividades com potencial causador de impacto ambiental. Essa pesquisa tem como objetivo estimar a vulnerabilidade natural à contaminação do Sistema Aquífero Serra Geral (SASG) no município de Panambi - RS, além de alocar as principais atividades com potencial risco à contaminação do meio subterrâneo.

\section{METODOLOGIA}

\subsection{Caracterização da área de estudo}

O município de Panambi está localizado na região Noroeste do Estado do Rio Grande do Sul, (Figura 1). O município possui uma população de 38.058 habitantes, sendo destes 3.496 residentes no meio rural e 34.562 habitantes residentes no meio urbano (IBGE, 2010).

Panambi destaca-se no cenário industrial gaúcho pelo seu significativo número de indústrias e seu elevado padrão gerencial e tecnológico. É hoje o terceiro polo metalmecânico do Estado. Neste município, encontramos centenas de pequenas, médias e grandes indústrias, que se dedicam aos mais variados ramos de atividade industrial, destacando-se as metalúrgicas, metalmecânicas, de eletroeletrônica, malharias, madeira, moveleira e de produtos alimentícios (GRACIOLI, 2012). No Município, se tem 221 atividades antrópicas cadastradas, com destaque a 38 indústrias de fabricação de materiais específicos, 14 postos de combustíveis e 2 aterros sanitários (FEPAM, 2019a). 
No que se refere à hidrografia, o município está inserido na Região Hidrográfica do Uruguai (U), Bacia Hidrográfica do Rio Ijuí (U - 90), conforme informações da SEMA (2004). Segundo FEPAM (2019b), as atividades econômicas desta bacia, de maneira geral, estão ligadas ao setor primário, predominando as lavouras de soja, porém ocorrem setores secundários e terciários em alguns municípios, sendo um destes Panambi - RS.

Em relação à geologia local, se tem a Formação Serra Geral (FSG), com predomínio das Fácies Gramado e em uma pequena porção, as Fácies Paranapanema, segundo CPRM (2006). A FSG é oriunda de derrames basálticos, com isso seus solos são oriundos de rochas basálticos e seus produtos de intemperização.

Nas características hidrogeológicas, o município está inserido no SASG I (MACHADO e FREITAS, 2005). Esses sistemas, segundo estes autores, apresentam produtividade alta a média para águas subterrâneas com porosidade por fraturas. Com isso, esse aquífero é do tipo cristalino fissural, coberto por rochas basálticas da FSG, apresentando recargas principalmente por fraturas das rochas (Freitas et al., 2012).

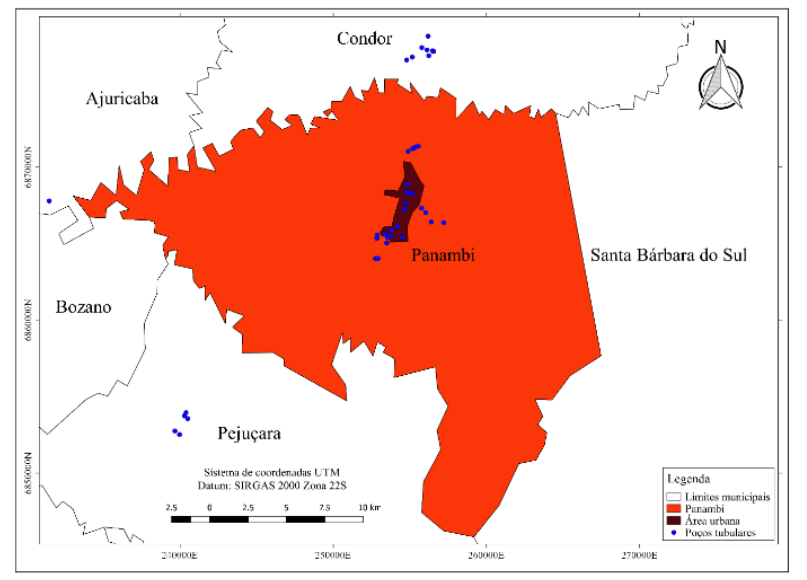

Figura 1 - Localização do município de Panambi-RS. Fonte: Construído a partir de IBGE (2005) e SIAGAS (2019).

\subsection{Obtenção das informações para estimativa da} vulnerabilidade natural do aquífero à contaminação

Para a estimativa da vulnerabilidade natural do aquífero à contaminação, foi utilizado o sistema GOD (Foster et al., 2002; 2006). O índice de vulnerabilidade é obtido a partir do produto das variáveis $\mathrm{G}, \mathrm{O}$ e $\mathrm{D}$, onde a vulnerabilidade é classificada como insignificante (valores entre 0 e 0,1$)$, baixa $(0,1$ e 0,3 ), média $(0,3$ e 0,5$)$, alta $(0,5$ e 0,7$)$ e extrema $(0,7$ e 1,0$)$.

A Figura 2 mostra o exemplo de aplicação do sistema GOD (Foster et al., 2002; 2006) na área de estudo.
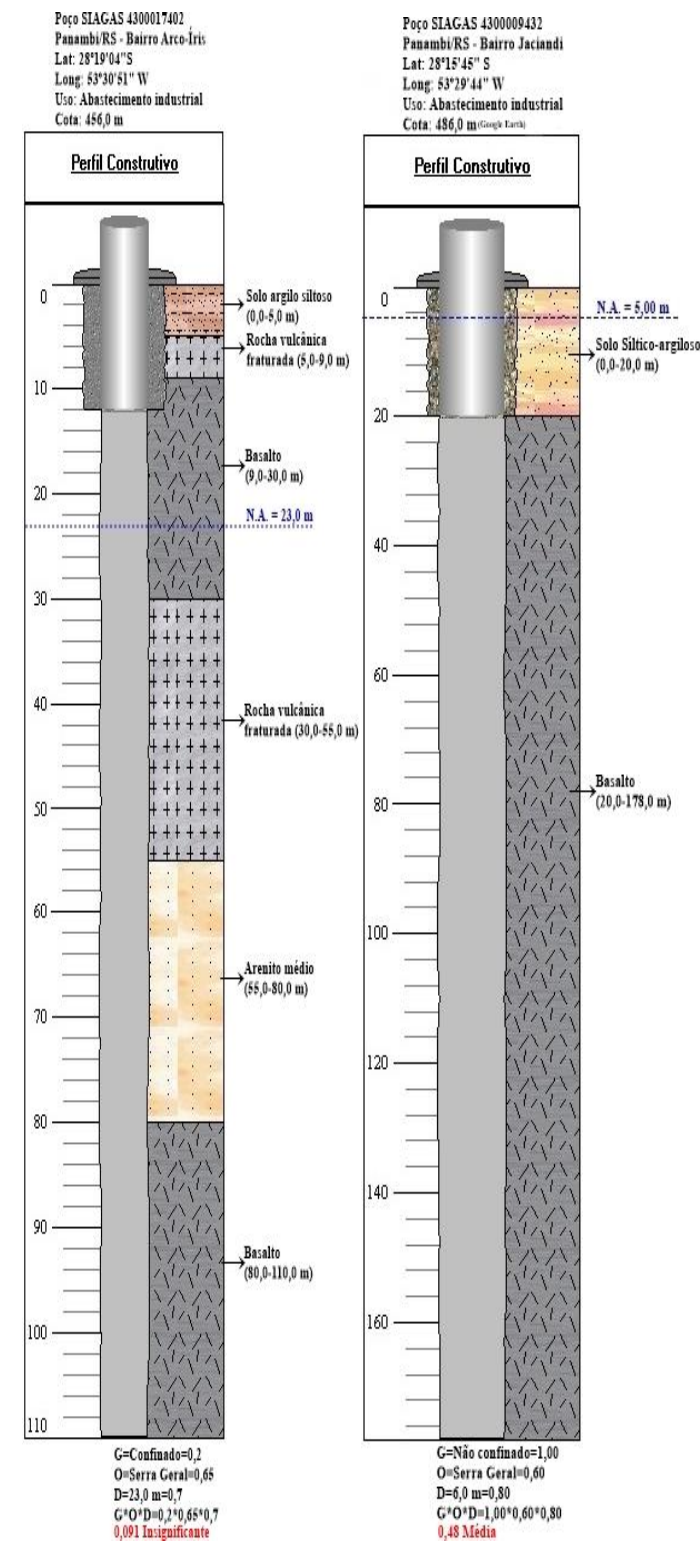

Figura 2 - Exemplo de aplicação do sistema GOD (Foster et al., 2002; 2006). Fonte: SIAGAS (2015).

As informações necessárias para aplicação do método foram obtidas no portal do Sistema de Informações de Águas Subterrâneas (SIAGAS), mantido pela Companhia de Pesquisa de Recursos Minerais (CPRM) do Sistema Geológico do Brasil, disponível

em: http://siagasweb.cprm.gov.br/layout/pesquisa_complexa.php. A partir disso, foram coletadas as informações de 68 captações subterrâneas entre os municípios de Condor, Cruz Alta, Panambi e Pejuçara, conforme proposto por Costa et al. (2011). Isso foi necessário pois as informações dos 21 poços cadastrados em Panambi não representarem a totalidade da área do município de Panambi, pois neste caso teria que ser realizada uma extrapolação 
dos dados, o que poderia ocasionar uma subjetividade dos resultados.

Essas informações foram analisadas dentro de um ambiente SIG (Sistema de Informações Geográficas). Foram utilizados os dados de coordenadas Universal Transversa de Mercator (UTM), nível estático da perfuração dos poços, perfis geológicos, cota da "boca" do poço e demais informações de interesse. Assim, foi possível estimar a vulnerabilidade natural à contaminação do SASG na escala de município de Panambi - RS. O Datum utilizado foi o South American Datum 1969 (SAD69), com uso do interpolador Inverse Distance Weighted (IDW).

\subsection{Determinação do uso do solo e identificação das fontes potenciais de contaminação}

As fontes potenciais de contaminação (Cemitérios, postos de combustíveis e indústrias tipo 3) foram identificadas a partir de imagens Google Earth Pro (Google, 2014) para o município de Panambi - RS. Essas fontes foram identificadas apenas na área urbana do município.

A partir da identificação dessas fontes, essas foram classificadas a partir do método POSH (Pollutant Origin and its Surcharge Hydraulically), proposto por Foster et al. (2002; 2006). Esse método classifica o grau de perigo que a atividade em questão oferece ao meio aquífero.

As imagens para determinação das classes de uso do solo foram obtidas no portal do United States Geological Servey (USGS) através do endereço: http://earthexplorer.usgs.gov/. As imagens são oriundas da missão Landsat 8 (USGS, 2016), datadas de 08 de fevereiro de 2016, sem a presença de nuvens. A partir dessas informações, o uso do solo foi classificado com as seguintes classes: água, área urbana, áreas agricultáveis e vegetação.

\section{RESULTADOS E DISCUSSÃO}

No município de Panambi as classes de uso de solo obtidas estão ilustradas na Figura 3, e seus respectivos percentuais na Tabela 1. Como pode ser observado, a maior classe de uso do solo observada foi as áreas de vegetação, com 163,25 km² (33,01\% da área municipal). A segunda classe mais representativa foi as áreas agricultáveis com $162,37 \mathrm{~km}^{2}(32,83 \%)$. A classe de solo exposto esteve presente em $155,40 \mathrm{~km}^{2}(31,44 \%)$, sendo que esta classe se relaciona com potenciais de erosão do solo. As demais classes de uso apresentaram menores proporções na área de Panambi, com $10,91 \mathrm{~km}^{2}(2,22 \%)$ de área urbana e $2,42 \mathrm{~km}^{2}(0,50 \%)$ de água.

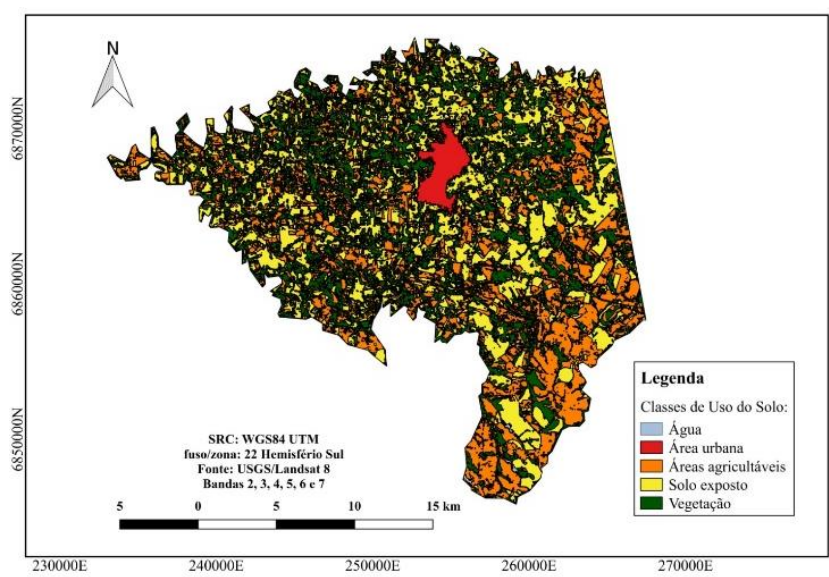

Figura 3 - Classes de uso do solo em Panambi - RS. Fonte: Construído a partir de IBGE (2005) e USGS (2016).

As classes de uso do solo possuem variados comportamentos quanto à infiltração de água no solo e consequente potencial de recarga do aquífero (Löbler et al., 2014). Segundo Tucci e Clarke (1997), a propensão de infiltração depende do uso e do tipo de solo. Normalmente, solos florestais apresentam boas condições de infiltração, tornando-os importantes fontes de abastecimento de água para os aquíferos (LIMA, 2008). Solos sem cobertura vegetal que sofrem com ações de compactação, tendem a reduzir drasticamente a capacidade de infiltração, resultando em maior escoamento superficial (TUCCI e CLARKE, 1997). Os mesmos autores afirmam que, a capacidade de infiltração varia com o tipo de solo e sua condição de umidade, como exemplo, um solo argiloso pode apresentar alta capacidade de infiltração quando estiver seco, porém, após receber umidade pode tornar-se quase que impermeável.

Assim, na área em estudo, ocorre um predomínio das classes de vegetação e agricultura. As áreas de agricultura podem apresentar problemas relacionados com à contaminação, não somente no meio superficial e/ou subterrâneo, pois essa atividade utiliza fertilizantes químicos e agrotóxicos. Assim, esses fertilizantes podem ser carreados aos recursos hídricos superficiais ou ainda infiltrar pelo perfil de solo e atingir o nível freático.

Hirata e Varnier (1998) dizem que os problemas relacionados à atividade agrícola incluem a salinização do solo e a contaminação do aquífero por pesticidas e nitratos. 
Tabela 1 - Percentuais das classes de uso do solo em Panambi RS. Fonte: Autores.

\begin{tabular}{c|c|c}
\hline Classe de uso do solo & Área $\left(\mathbf{k m}^{2}\right)$ & Área $(\%)$ \\
\hline Vegetação & 163,25 & 33,01 \\
\hline Áreas agricultáveis & 162,37 & 32,83 \\
\hline Solo exposto & 155,40 & 31,44 \\
\hline Área urbana & 10,91 & 2,22 \\
\hline Água & 2,42 & 0,50 \\
\hline Total & $\mathbf{4 9 4 , 3 5}$ & $\mathbf{1 0 0 , 0 0}$ \\
\hline
\end{tabular}

A Figura 4 mostra a estimativa da vulnerabilidade natural à contaminação no Município de Panambi, a partir do uso do sistema GOD (Foster et al., 2002; 2006). Conforme ilustrado, as classes de vulnerabilidade variaram de insignificante (classe predominante na área do município) à baixa. O predomínio dessas menores classes de vulnerabilidade pode estar relacionado, principalmente pelas Formações Geológicas existentes, no caso, as rochas vulcânicas da Formação Serra Geral e seus produtos de intemperização, segundo CPRM (2006). Porém, deve-se se ter atenção com o fraturamento das rochas basálticas, as quais podem favorecer a infiltração do contaminante e contaminar o aquífero confinado. Visto que esse tipo de aquífero apresenta suas zonas de recarga por fraturas das rochas basálticas (Freitas et al., 2012).

Diversos estudos foram realizados nesses tipos de geologia e hidrologia (Silvério da Silva et al., 2013; Reginatto e Alhert, 2013; Lobler e Silvério da Silva, 2015; Borba et al., 2016; Terra et al., 2016; Fernandes et al., 2016; Borges et al., 2017; Favaretto et al., 2020), os quais apresentaram resultados satisfatórios (Tabela 2). Como pode ser observado, nas pesquisas realizadas no SASG ocorreu o predomínio das menores classes de vulnerabilidade, sendo essa condição também obtida nessa pesquisa.

Tabela 2 - Classes de vulnerabilidade pelo método GOD no SASG em outras regiões, com mesma hidrologia e geologia. Fonte: Autores.

\begin{tabular}{c|c|c}
\hline Autores & $\begin{array}{c}\text { Classes de } \\
\text { vulnerabilidade }\end{array}$ & $\begin{array}{c}\text { Predomínio de } \\
\text { classes }\end{array}$ \\
\hline $\begin{array}{c}\text { Silvério da Silva et al. } \\
(2013)\end{array}$ & $\begin{array}{c}\text { Insignificante a } \\
\text { média }\end{array}$ & $\begin{array}{c}\text { Média } \\
(85,50 \%)\end{array}$ \\
\hline $\begin{array}{c}\text { Reginato e Alhert } \\
(2013)\end{array}$ & Baixa a média & Baixa (79,00\%) \\
\hline $\begin{array}{c}\text { Lobler e Silvério da } \\
\text { Silva (2015) }\end{array}$ & $\begin{array}{c}\text { Insignificante a } \\
\text { extrema }\end{array}$ & $\begin{array}{c}\text { Média } \\
(30,76 \%)\end{array}$ \\
\hline Borba et al. (2016) & $\begin{array}{c}\text { Insignificante a } \\
\text { alta }\end{array}$ & $\begin{array}{c}\text { Insignificante } \\
(73,15 \%)\end{array}$ \\
\hline Borges et al. (2017) & Baixa a extrema & Baixa $(66,21 \%)$ \\
\hline Favaretto et al. (2020) & $\begin{array}{c}\text { Insignificante a } \\
\text { baixa }\end{array}$ & Baixa $(86,46 \%)$ \\
\hline
\end{tabular}

Ainda na Figura 4, estão ilustradas as principais fontes (Cemitérios, postos de combustíveis e indústrias tipo 3) com potencial gerador de carga contaminante, apresentando risco à contaminação do aquífero a partir do método POSH (Foster et al.,
2002; 2006) na área urbana do município. Como pode ser observado, as fontes foram classificadas em reduzido (Cemitérios), moderado (Postos de combustíveis) e elevado (Indústrias tipo 3: Processamento de metais) potencial de carga contaminante no subsolo.

Como pode ser observado na Figura 4, na parte norte do mapa, os cemitérios que são caracterizados por apresentarem reduzido potencial de geração de contaminantes, juntamente com a indústria tipo 3, que apresentam elevado risco potencial de geração de contaminantes segundo Foster et al. (2002; 2006), estão localizadas nas áreas de baixa vulnerabilidade. Sendo que as demais atividades estão alocadas nas áreas classificadas como de insignificante vulnerabilidade. Embora essas atividades (cemitérios no caso) apresentem reduzido risco (Foster et al., 2002; 2006), por estar localizada em uma área de vulnerabilidade baixa, apresentam risco à contaminação do aquífero. Já as indústrias tipo 3, apresentam elevado risco e estão localizadas nas regiões de baixa vulnerabilidade natural a contaminação do aquífero.

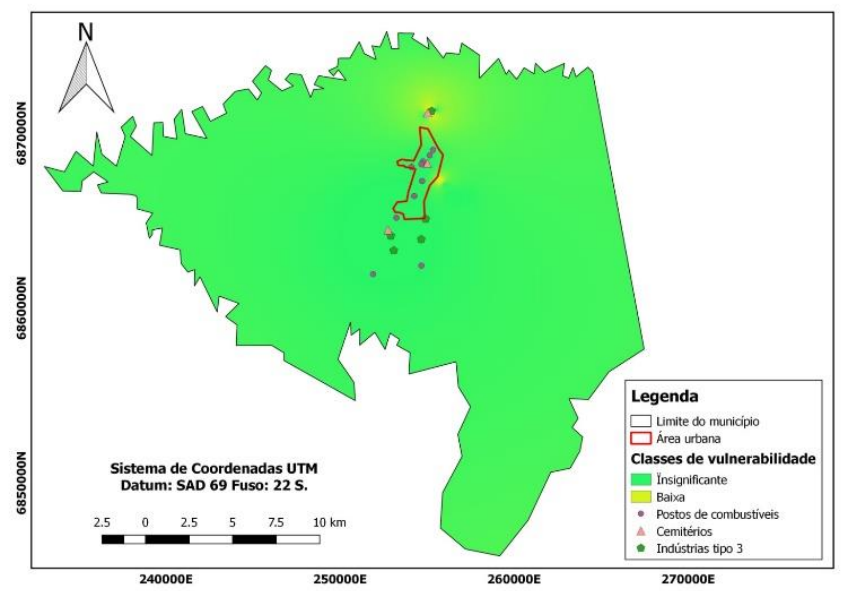

Figura 4 - Principais fontes com potencial gerador de carga contaminante. Fonte: Construído a partir de IBGE (2005) e SIAGAS (2016), utilizando o sistema GOD.

\section{CONSIDERAÇÕES FINAIS}

A partir desse estudo, foi possível se analisar que o município de Panambi-RS, localizado nas rochas vulcânicas da Formação Serra Geral apresentou as menores classes de vulnerabilidade à contaminação: Insignificante e baixa. Isso, pode estar relacionado a presença dessas rochas, que na maioria das vezes proporciona uma característica confinada ao meio. Além disso, as atividades com potenciais riscos de contaminação estão alocadas nas classes de menor vulnerabilidade.

Assim, esses estudos apresentam relativa importância ao meio, pois indicam quais as áreas mais vulneráveis à contaminação. Deste modo, podem servir de base para elaboração de planos diretores municipais. 


\section{REFERÊNCIAS}

ALLER, L.; BENNETT, T.; LEHR, J. H.; PETTY, R. J. DRASTIC: A standardized system for evaluating ground water pollution potential using hydrogeologic settings. Chicago: NWWA/EPA, 1987.

BORBA, W. F.; FERNANDES, G. D. A.; TERRA, L. G.; LOBLER, C. A.; SILVÉRIO DA SILVA, J. L. Assessment of the Intrinsic Vulnerability to Contamination of the Aquifer in River Basin Passo Fundo of Rio Grande do Sul. Anuário do Instituto de Geociências (UFRJ. Impresso), v. 39, 145-154. Rio de Janeiro: 2016. http://dx.doi.org/10.11137/2016_2_145_154

BORGES, V. M.; ATHAYDE, G. B.; REGINATO, ROEHE, P. A. Avaliação da vulnerabilidade natural à contaminação do sistema aquífero Serra Geral no Estado do Paraná - Brasil. Águas Subterrâneas, v. 31, 327-337, 2017. https://doi.org/10.14295/ras.v31i4.28857

CPRM - COMPANHIA DE PESQUISA DE RECURSOS MINERAIS. Mapa geológico do Rio Grande do Sul, escala 1:750.000. CPRM: Brasília, 2006.

CPRM - COMPANHIA DE PESQUISA DE RECURSOS MINERAIS. SIAGAS. Sistema de informações de águas subterrâneas. Disponível em: http://siagasweb.cprm.gov.br/layout/. Acesso em: 20/05/2015.

COSTA, M. L. M. C.; COSTA, M. M. R. R.; REGO, J. C. R.; ALBURQUERQUE, J. do P. T. Preposição de critérios de outorga para águas subterrâneas. Revista Brasileira de Recursos Hídricos, v. 16, n. 1, 105-113. Porto Alegre: 2011. https://doi.org/10.21168/rbrh.v16n1.p105-113

CUTRIM, A. O.; CAMPOS, J. E. G. Avaliação da vulnerabilidade e perigo à contaminação do Aquífero Furnas na cidade de Rondonópolis (MT) com aplicação dos métodos GOD e POSH. Geociências (UNESP. Impresso), v. 29, 401-411. Rio Claro: 2010.

ENCINA, C. C. C.; MARQUES, M. R.; DIODATO, M. A.; PEREIRA, L. E.; ALBREZ, E. A. OLIVEIRA, A. P. G.; MIOTO, C. L.; MIRANDA, V. R.; MIRANDA, L. M.; COELHO, L. S.; DALMAS, F. B.; FILHO, A. C. P. Geotechnology Applied to the Environmental Analysis of the Olho d'Água River Watershed, Municipality of Jardim, Mato Grosso do Sul - Brazil. Anuário do Instituto de Geociências - UFRJ, v. 41, n. 2, 577-584. Rio de Janeiro: 2018. http://dx.doi.org/10.11137/2018_2_577_584

FAVARETTO, J. R.; ALLASIA, D. G.; SILVÉRIO DA SILVA, J. L.; BORBA, W. F.; ROSA, C. N. ; FERNANDES, G. D. Estimativa da Susceptibilidade à Contaminação do Sistema Aquífero Serra Geral em Aratiba - RS por Diferentes Métodos. RBC. REVISTA BRASILEIRA DE CARTOGRAFIA (ONLINE), v. 72, 1-18, 2020. https://doi.org/10.14393/rbcv72n1-47184
FUNDAÇÃO ESTADUAL DE PROTEÇÃO AMBIENTAL LUIZ CARLOS ROESSLER (FEPAM). (2019a). Licenciamento Ambiental. FEPAM. Disponível em: <http://www.fepam.rs.gov.br/licenciamento/Area1/default. asp>. Acesso em: 29 out. 2019.

FUNDAÇÃO ESTADUAL DE PROTEÇÃO AMBIENTAL LUIZ CARLOS ROESSLER (FEPAM). (2019b). U90 Panambi. FEPAM. Disponível em: <http://www.fepam.rs.gov.br/qualidade/bacia_uru_panambi .asp>. Acesso em: 29 out. 2019.

FERNANDES, G. D. A.; BORBA, W. F.; LASTA, L.; LOBLER, C. A.; SILVÉRIO DA SILVA, J. L. Uso do sistema GOD para determinação da vulnerabilidade natural do aquífero à contaminação em Marau - RS. In $5^{\circ}$ Congresso Internacional de Tecnologias para o Meio Ambiente. Anais... Bento Gonçalves, $5^{\circ}$ Congresso Internacional de Tecnologias para o Meio Ambiente, 2016.

FOSTER, S.; HIRATA, R.; GOMES, D.; D'ELIA, M.; PARIS, M. Groundwater quality protection: a guide for water utilities, municipal authorities, and environment agencies. 1 ed. Washington: World Blank, 2002. 114p.

FOSTER, S.; HIRATA, R.; GOMES, D.; D'ELIA, M.; PARIS, M. Proteção da qualidade da água subterrânea: um guia para empresas de abastecimento de água, órgãos municipais e agências ambientais. 1 ed. São Paulo: Servmar, 2006. 114p.

FRANCISCO, P. R.; RIBEIRO, G. N.; SILVINO, G. S.; PEREIRA, F. C.; NETO, J. M. M.; SILVA, V. M. A. Geotecnologias aplicada a estudos ambientais. E-book.

FREEZE, A. R.; CHERRY, J. A. Groundwater. Traduzido por Everton de Oliveira (Coord). São Paulo: Everton de Oliveira, 2017. 681p.

FREITAS, M. A.; BINOTTO, R. B.; NANNI, A. S.; RODRIGUEZ, A. L. M.; BORTOLI, C. R. Avaliação do potencial hidrogeológico, vulnerabilidade intrínseca e hidroquímica do Sistema Aquífero Serra Geral no noroeste do Estado do Rio Grande do Sul. Revista Brasileira de Recursos Hídricos, Ano 2012, v. 17, p. 31-41. Porto Alegre, 2012. http://dx.doi.org/10.21168/rbrh.v17n2.p31-41

GOOGLE. Google Earth. Version Pro. 2016. Imagens do município de Panambi - RS. Disponível em: https://www.google.com.br/earth/download/gep/agree.html. Acesso em: 06/07/2016.

GRACIOLI, D. J. F. A cidade de Panambi e as transformações recentes na configuração territorial. Disponível em: http://bibliodigital.unijui.edu.br:8080/xmlui/bitstream/hand le/\%20123456789/1407/. Acesso em 15/06/2016.

HIRATA, R.; VARNIER, C. Águas subterrâneas e agronegócios. In X Congresso Brasileiro de Águas Subterrâneas, Aanais... São Paulo, X Congresso Brasileiro de Águas Subterrâenas, v. 1, 397-412. 1998. 
INSTITUTO BRASILEIRO DE GEOGRAFIA E ESTATÍSTICA (IBGE). Malha municipal digital do Brasil: 2005 (escala 1:500.000). Rio de Janeiro: Instituto Brasileiro de Geografia e Estatística, 2005. Disponível em: ftp://geoftp.ibge.gov.br/mapas/malhas_digitais/municipio_ 2005/E1000/Proj_Geografica/Arc View_shp/Regiao/Sul/. Acesso em: 20/05/2015.

INSTITUTO BRASILEIRO DE GEOGRAFIA E ESTATÍSTICA (IBGE). Censo demográfico 2010. Disponível em: https://censo2010.ibge.gov.br/. Acesso em: 06/07/2016.

LIMA, W. P. Hidrologia Florestal Aplicada ao Manejo de Bacias Hidrográficas. 2 ed. Piracicaba: Universidade de São Paulo, 2008. $253 \mathrm{p}$.

LÖBLER, C. A.; TERRA, L. G.; SILVÉRIO DA SILVA, J. L. Mapeamento da vulnerabilidade das águas subterrâneas e uso do solo na área urbana do município de Nova Palma, RS. Ciência e Natura, v. 36, 587-592. Santa Maria: 2014. https://doi.org/10.5902/2179460X14843

LOBLER, C. A.; SILVÉRIO DA SILVA, J. L. Vulnerabilidade à contaminação das águas subterrâneas do município de Nova Palma, Rio Grande do Sul, Brasil. Revista Ambiente \& Água, v. $10,141-152,2015$. https://doi.org/10.4136/ambiagua. 1390

MACHADO, J. L. F.; FREITAS, M. A. Projeto mapa hidrogeológico do Estado do Rio Grande do Sul: escala 1:750.000, relatório final. Porto Alegre: CPRM. 2005.

NANNI, A. S.; FREITAS, M. A.; TEDESCO, M. A.; BINOTTO, R. B. Vulnerabilidade natural e risco de contaminação do aquífero Serra Geral pela suinocultura na região das missões - RS. In XVI Simpósio Brasileiro de Recursos Hídricos, Anais... João Pessoa, XVI Simpósio Brasileiro de Recursos Hídricos. 2005.

REGINATO, P. A. R.; AHLERT, S. Vulnerabilidade do Sistema Aquífero Serra Geral na Região Nordeste do Estado do Rio Grande do Sul. Águas Subterrâneas, v. 27, 32-46. São Paulo: 2013. https://doi.org/10.14295/ras.v27i2.27060

RIBEIRO, D. D. M.; ROCHA, W. J. S. F.; GARCIA, A. J. V. Vulnerabilidade natural à contaminação dos aquíferos da sub-bacia do Rio Siriri, Sergipe. Revista Águas Subterrâneas, $\quad$ v. $\quad 25, \quad 91-102, \quad 2011$. https://doi.org/10.14295/ras.v25i1.19366

SECRETARIA DO MEIO AMBIENTE DO ESTADO DO RIO GRANDE DO SUL Mapa das Bacias Hidrográficas e Municípios do Rio Grande do Sul. Porto Alegre: SEMA. 2004.

SILVÉRIO DA SILVA, J. L.; DESCOVI FILHO, L. L. V.; LORENSI, R. P.; CRUZ, J. C.; ELTZ, F. L. Vulnerabilidade do Aquífero Serra Geral à contaminação no município de Erechim - Rio Grande Do Sul - Brasil.
Ciência e Natura, Ano 2013, v. 35, p. 10-23. Santa Maria: 2013. https://doi.org/10.5902/2179460X9598

TERRA, L. G.; BORBA, W. F.; FERNANDES, G. D. A.; TROMBETA, H. W.; SILVÉRIO DA SILVA, J. L. Caracterização hidroquímica e vulnerabilidade natural à contaminação das águas subterrâneas no município de Ametista do Sul - RS. Revista Monografias Ambientais, Ano 2016, v. 15, p. 94-104. Santa Maria: 2016. https://doi.org/10.5902/2236130820033

TUCCI, C. E. M.; CLARKE, R. T. Impacto das mudanças da cobertura vegetal no escoamento: revisão. Revista Brasileira de Recursos Hídricos, Ano 1997, v. 2, n. 1, p. 135-152. Porto Alegre: 1997. https://doi.org/10.21168/rbrh.v2n1.p135-152

UNITED STATES GEOLOGICAL SERVEY - USGS. Shuttle Radar Topography Mission, 1 Arc Second scene SRTM1S28W054V3, Unfilled Unfinished 2.0, Global Land Cover Facility, University of Maryland, College Park, Maryland. Disponível em: https://earthexplorer.usgs.gov/. Acesso em: 15/02/2016.

\section{AGRADECIMENTOS}

Os autores agradecem a CAPES/FAPERGS e CAPES/CNPq pelo apoio financeiro conedido por meio de bolsas de PósGraduação do PPGEC/UFSM e PPGEF/UFSM.

Recebido em: 25/10/2019

Aceito para publicação em: 06/08/2020 